\title{
Stiff Left Atrium Syndrome After Atrial Fibrillation Ablation - A Diagnosis Not To Forget
}

Raul Serra Valério ${ }^{1, *}$, Rafael Thiesen Magliari², Alfredo Augusto Eyer Rodrigues ${ }^{1,3}$, Cristiano de Oliveira Dietrich ${ }^{2,3}$

\section{ORCID ID}

Valerio RS (1) https://orcid.org/0000-0003-1530-3795

Rodrigues AAE (1) https://orcid.org/0000-0001-5885-6715

Magliari RT (1) https://orcid.org/0000-0002-3839-6253

Dietrich CO (D) https://orcid.org/0000-0002-7373-9119

\begin{abstract}
Atrial fibrillation is the most prevalent arrhythmia in clinical practice and has different strategies for its control. Of these strategies, the percutaneous ablation of the pulmonary veins stands out, with robust results in relation to drug treatment. It is an invasive procedure and, therefore, not free from complications, which must be properly diagnosed and treated. Among the possible complications, there is stiff atrium syndrome, characterized by reduced atrial compliance caused by post-ablation fibrosis, which, in turn, leads to atrial filling dysfunction and the consequent increase in atrial and venous capillary pulmonary pressures. The case report demonstrates this infrequent but important complication, which presents good results for clinical treatment, in addition to the contribution of cardiac magnetic resonance in its diagnosis and in the assessment of arrhythmia recurrence rates.
\end{abstract}

KEYWORDS: Atrial fibrillation; Catheter ablation; Stiff left atrium; Magnetic resonance imaging.

\section{INTRODUCTION}

Atrial fibrillation (AF) is the most prevalent sustained arrhythmia in clinical practice. Often, drug treatment with antiarrhythmics is not enough to maintain sinus rhythm, which makes percutaneous ablation of atrial fibrillation (PAAF), using the technique of antral isolation of the pulmonary veins (PV), a therapy already established in the control of rhythm ${ }^{1}$. As it is an invasive strategy, there are infrequent complications that can occur intra-procedurally, or in the first 30 days after surgery. Stiff left atrium syndrome (SLAS), an entity little remembered in clinical practice and with

1. DASA - São Paulo (SP), Brazil.

2. Hospital do Coração - São Paulo (SP), Brazil.

3. Universidade Federal de São Paulo - São Paulo (SP), Brazil.

*Corresponding author: raulsvalerio@gmail.com

Received: Jun 21, 2021 | Accepted: Nov 6, 2021 
poorly understood pathophysiology, is an important complication after PAAF, characterized by the presence of dyspnea and pulmonary hypertension secondary to loss of atrial compliance in the absence of mitral regurgitation and stenosis PV, in addition to secondary factors. SLAS has an incidence of 1.4-8\% and may occur in the first three months post-ablation or with an acute onset after surgery ${ }^{2,3}$.

The aim of this report was to present a rare case in which the patient developed SLAS early after ablation of persistent long-term AF, with demonstration of the diagnostic and therapeutic methodology.

\section{CLINICAL CASE}

A 57-year-old male patient was referred for catheter ablation of symptomatic AF in August 2020. He had been diagnosed with AF for three years (Fig. 1) and had failed rhythm control attempts with propafenone, amiodarone and electrical cardioversion. Despite the use of $50 \mathrm{mg}$ /day metoprolol associated with $200 \mathrm{mg} /$ day amiodarone to control heart rate, he persisted with limited functional capacity (class III EHRA), in addition to accelerated palpitations at rest. Previous history of smoking (he stopped 12 years ago) and moderate alcohol consumption. He used irregular antithrombotic therapy with acetylsalicylic acid (ASA) $100 \mathrm{mg}$ daily. In the first evaluation by the electrophysiologist, it was decided to withdraw the ASA and introduce oral anticoagulation with rivaroxaban $20 \mathrm{mg}$ daily.

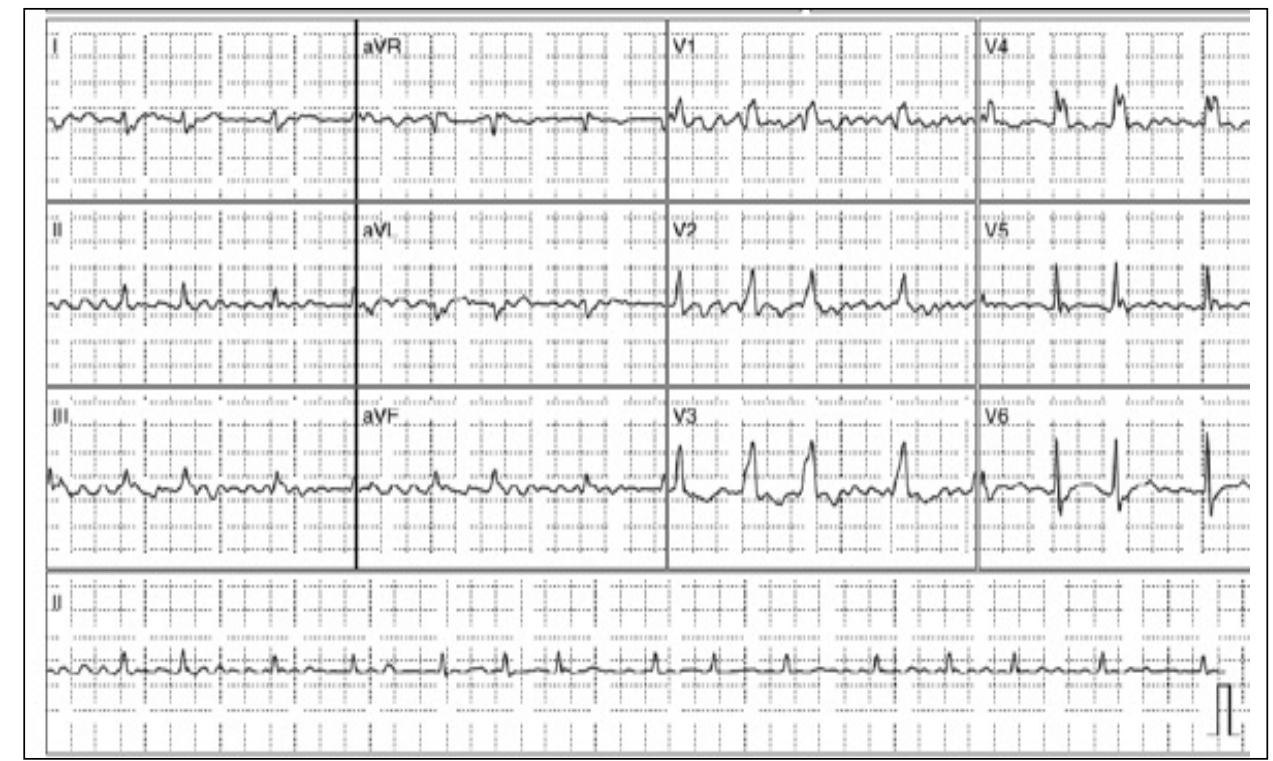

Figure 1. Electrocardiogram showing atrial fibrillation rhythm and right bundle-branch block.

After four weeks of oral anticoagulation, a transesophageal echocardiogram was performed, which showed the presence of spontaneous contrast grade III/IV and a reduced rate of emptying of the left atrial appendage, without the presence of an intracavitary thrombus. In December 2020, catheter ablation was performed under general anesthesia. Through a puncture in the left femoral vein, an intracardiac echocardiogram (ICE) catheter was inserted. Three punctures in the right femoral vein were performed to insert the electrophysiology catheters into the cardiac chambers. Vascular punctures were guided by a vascular echo doppler.

Full anticoagulation with intravenous heparin (initial dose 10,000 IU and additional 1,000-5,000 IU) was instituted immediately after the end of venous punctures, with activated coagulation time control being performed every 30 min, with the aim of maintaining this time above $350 \mathrm{~s}$. The decapolar catheter was inserted into the coronary sinus using ICE images and recording of local atrioventricular electrograms. Then, two sequential transseptal punctures were performed under ICE guidance without the use of fluoroscopy. Through the transseptal sheaths (Preface and Mobicath), the circular 
Lasso-Nav and Thermocool-SmartTouch SF therapeutic catheters were inserted into the left atrium. Using the Carto-3 electroanatomical system, the three-dimensional geometry of the left atrium and PV was constructed (Fig. 2).

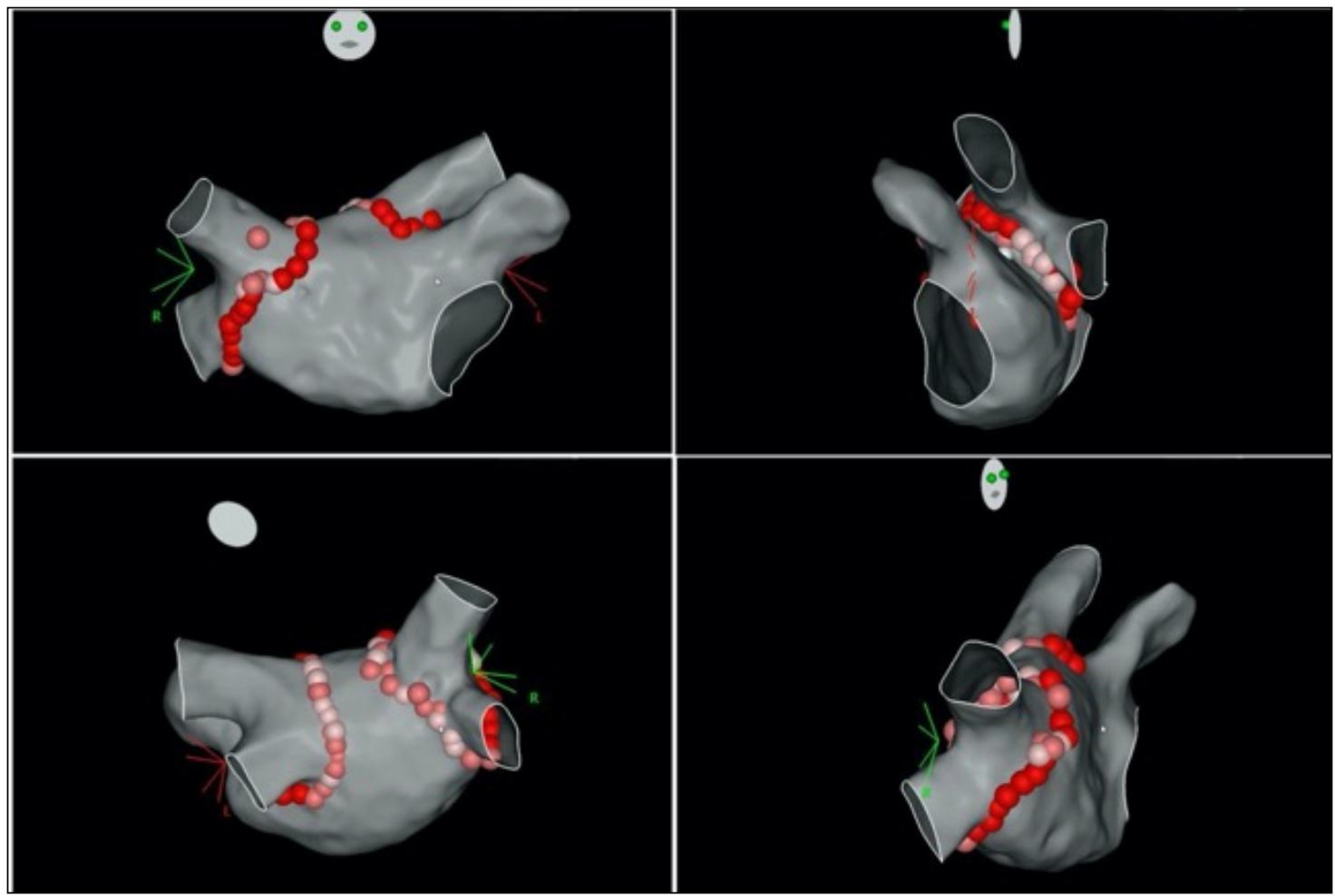

Figure 2. Radiofrequency catheter ablation for the treatment of atrial fibrillation. Isolation of the four pulmonary veins by circumferential antral ablation (point-to-point radiofrequency) guided by three-dimensional electroanatomical mapping (CARTO 3 system, Biosense Webster - J\&J, Diamond Bar, CA).

Voltage map during AF demonstrated the absence of low voltage areas $(<0.05-0.20 \mathrm{mV})$ in the left atrium. First, antral isolation of the left PVs was performed by point-to-point radiofrequency applications, with fixed energy of $40 \mathrm{~W}$, irrigation of $15 \mathrm{~mL} / \mathrm{min}$, aiming at contact $>10 \mathrm{~g}$ and ablation index of 550 on the anterior wall, 450 on the ceiling and 350-400 on the back wall. Then, antral isolation of the right PVs was performed with the same parameters described above. During isolation of the right veins, sinus rhythm was restored. Pulmonary vein isolation was performed with the creation of a single block line ("first pass isolation") bilaterally, without the need for additional applications along the line.

After isoproterenol infusion, frequent atrial extrasystoles were recorded, whose mapping showed its origin in the superior vena cava (SVC), which justified its electrical isolation. Procedure performed uneventfully and using the zero fluoroscopy technique. Sixty-one radiofrequency (RF) applications were performed (32 on the right and 29 on the left), with a total time of radiofrequency application in the left atrium of $908 \mathrm{~s}$, with $488 \mathrm{~s}$ in the right veins and $420 \mathrm{~s}$ in the left veins. At the end of the procedure, the PV and SVC were evaluated with the aid of the ICE with demonstration of preserved diameters and flows. The esophageal thermometer was not used in this case. Patient was discharged from hospital $24 \mathrm{~h}$ after the procedure.

Two days after discharge, the patient developed edema in the lower limbs, tiredness (worsening of the functional class, NYHA III) and weight gain of approximately $5 \mathrm{~kg}$, justifying hospitalization for investigation. Still in the emergency room, venous Doppler ultrasound of the lower limbs and angiotomography (CT angiography) of the chest were performed, which ruled out deep vein thrombosis and pulmonary thromboembolism, respectively. Chest tomography (CT) showed signs of pulmonary congestion and moderate pleural effusion on the right (Fig. 3). Serial serum troponin levels were within normal limits. Laboratory evaluation of atrial natriuretic peptide (NT-PROBNP) at entry showed elevated levels of 2,770 pg/mL (reference less than $300 \mathrm{pg} / \mathrm{mL}$ ). Echocardiogram (ECHO) analysis was indicative of moderate pulmonary hypertension and estimated right ventricular systolic pressure of $50 \mathrm{mmHg}$. 


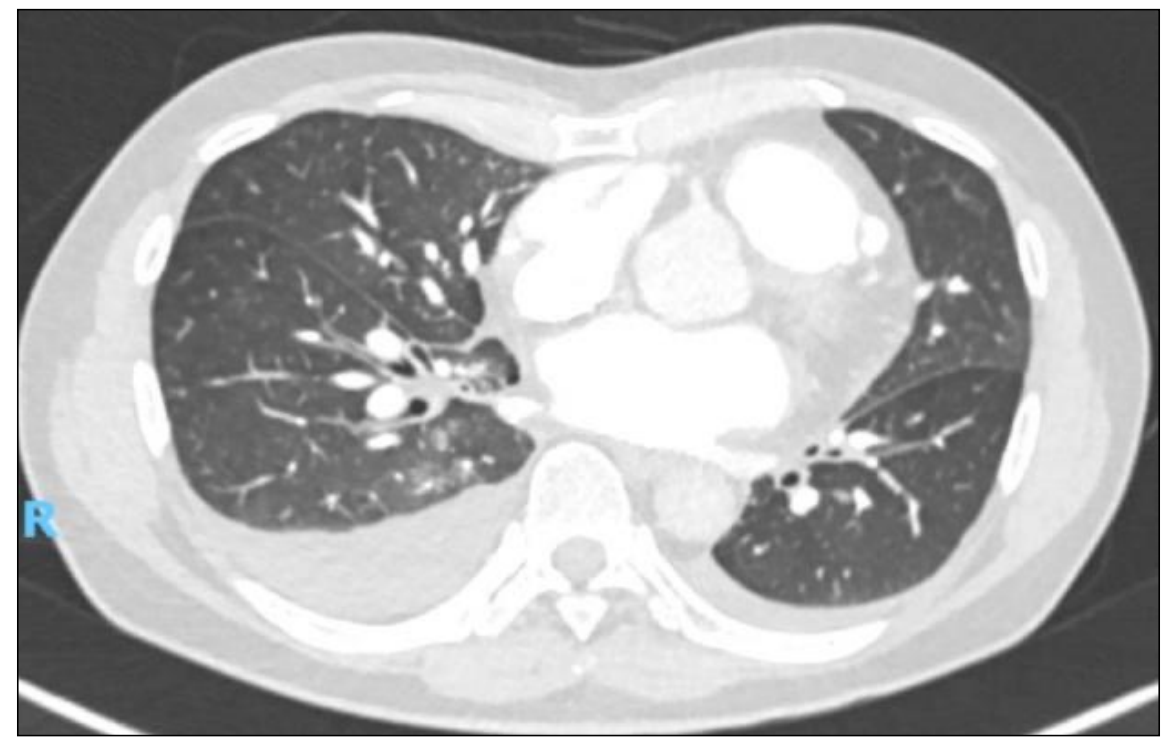

Figure 3. Chest tomography showing signs suggestive of pulmonary congestion and moderate pleural effusion on the right.

Initially, treatment with $25 \mathrm{mg}$ captopril and $1 \mathrm{mg} / \mathrm{kg}$ furosemide was instituted, with clinical improvement. Complementary investigation through coronary and left atrium CT angiography did not visualize PV stenosis or coronary lesions (Fig. 4), however the imaging exam was suggestive of left atrial systolic dysfunction.
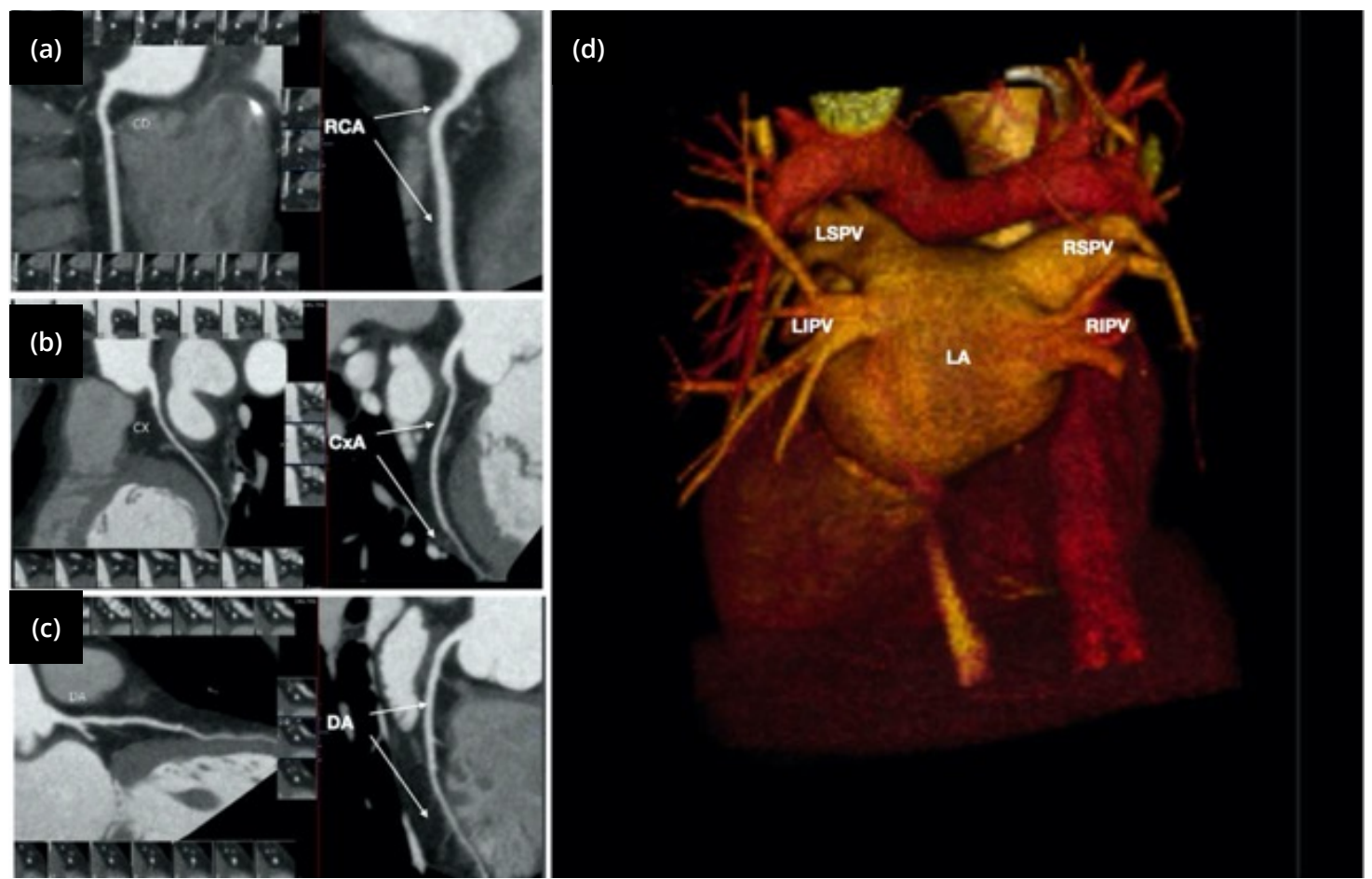

LSPV: left superior pulmonary vein; LIPV: left inferior pulmonary vein; RSPV: right superior pulmonary vein; RIPV: right inferior pulmonary vein.

Figure 4. Cardiac CT angiography. Sectional sections of the coronary arteries demonstrating absence of luminal reduction: (a) right coronary artery (RCA); (b) circumflex artery (CXA); (c) anterior descending artery without the presence of atherosclerotic plaques or luminal reduction; (d) three-dimensional tomographic reconstruction of the left atrium (LA) and pulmonary veins. Note the absence of pulmonary vein stenosis after ablation of atrial fibrillation.

Cardiac magnetic resonance imaging showed left atrium with preserved dimensions, with end diastolic volume of $46 \mathrm{~mL}$ and ejection volume of $12 \mathrm{~mL}$, signs of prolonged diastolic time and left atrial ejection fraction estimated 
at 25\%, in addition to the presence of late atrial enhancement (Fig. 5 and Videos 1 and 2 in Supplementary Data). In the evaluation by magnetic resonance angiography, no obstructions in the flow of pulmonary veins were evidenced (Fig. 6).
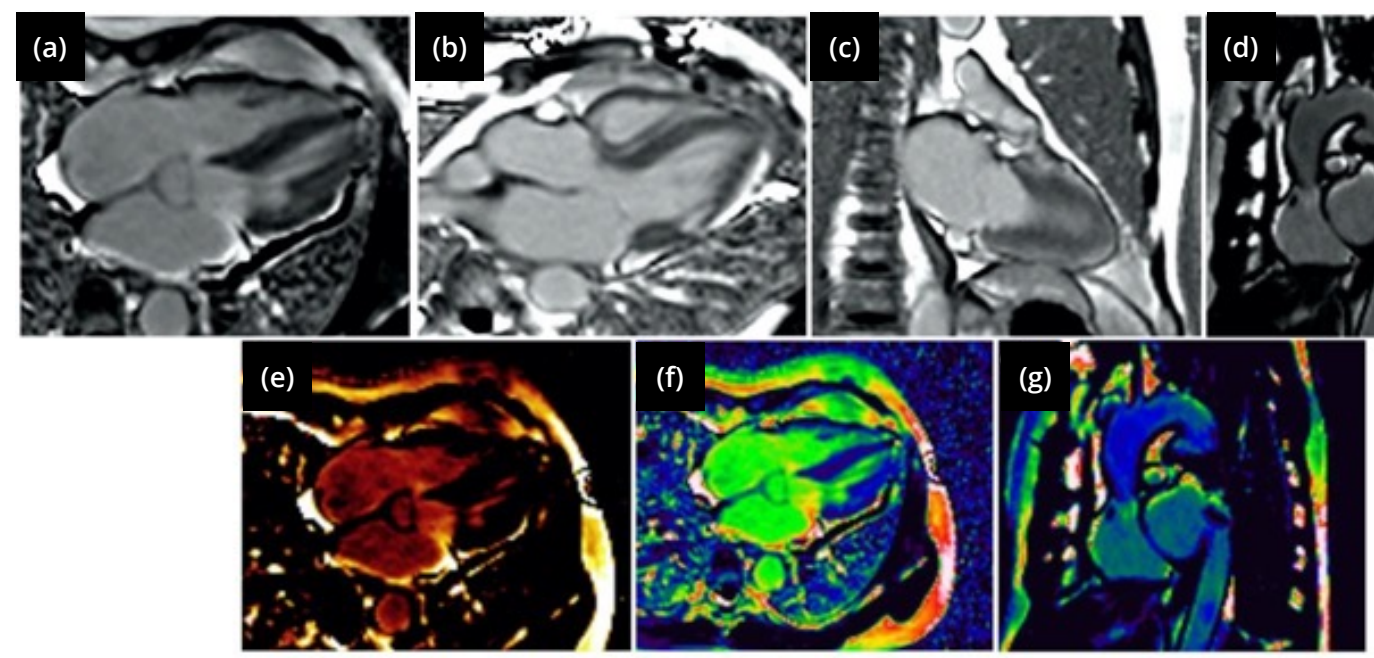

Figure 5. Cardiac resonance of the region of fibrosis in the left atrium demonstrated by the delayed enhancement technique, close to the regions of ablation in the antrum of the pulmonary veins. Four-chamber cardiac image $(a, b$, e and $f$ ) long axis, (c) bicameral long axis and ( $d$ and $g$ ) atrial short axis.
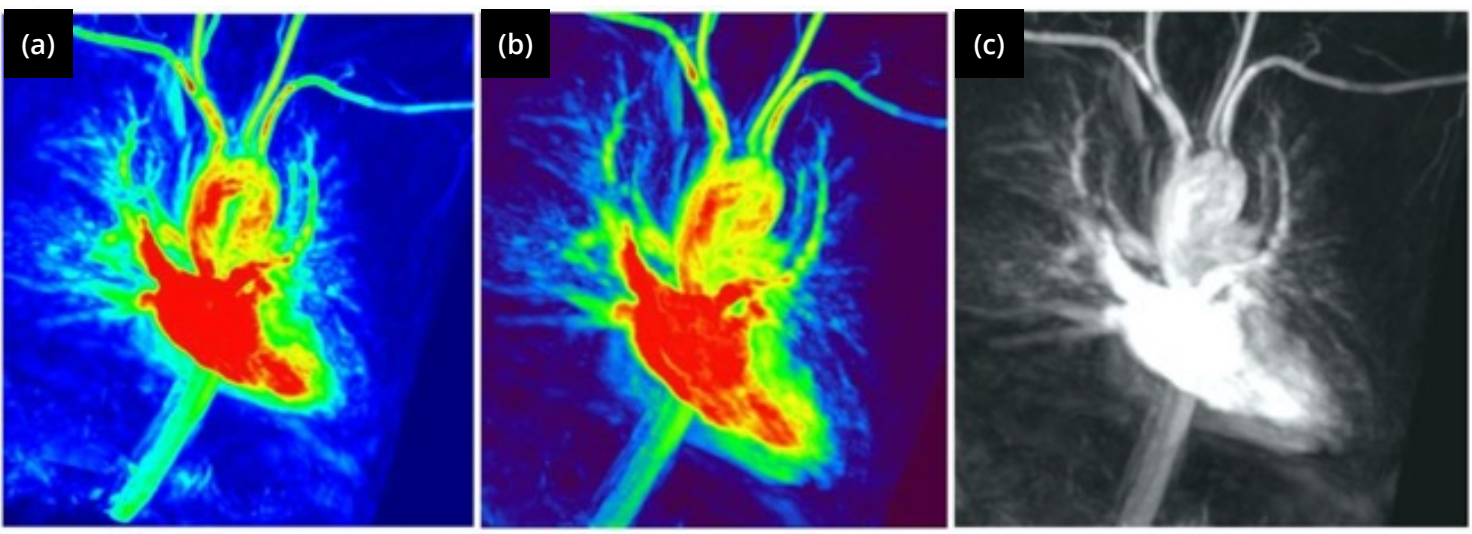

Figure 6. Cardiac MRI showing the flow preserved through the pulmonary veins.

After three weeks of drug treatment with captopril and a diuretic, the patient evolved with a significant improvement in his clinical condition and returned to NYHA functional class I. Laboratory tests demonstrated normalization of brain natriuretic peptide (BNP) levels. Then, diuretic and vasodilator therapy was suspended.

During the six-month clinical follow-up, the patient was asymptomatic and in NYHA functional class I, maintaining regular sinus rhythm without the need for antiarrhythmic therapy (discontinued after three months of the procedure). The echocardiogram indicated normalization of pulmonary artery pressure. Despite the CHA2DS2-VASc score of zero, it was decided to maintain oral anticoagulation due to diffuse left atrial hypokinesia.

\section{DISCUSSION}

Currently, catheter ablation for the treatment of $\mathrm{AF}$ is a well-established and widely used strategy to maintain sinus rhythm in symptomatic patients ${ }^{1}$. It has a high rate $(>80 \%)$ of rhythm control in experienced centers and a low incidence of complications, up to $4.5 \% \%^{1,4}$. 
The case report presents SLAS, an uncommon but potential complication of catheter ablation for the treatment of AF. Its incidence is $1.4-8 \%{ }^{2}$. The syndrome is characterized by decreased left atrial compliance and increased atrial diastolic pressure, with or without changes in atrial systolic function, which can be hemodynamically demonstrated by a deep $\mathrm{V}$ wave of the venous pulse, in the absence of significant mitral regurgitation ${ }^{5-8}$.

The increase in left atrial pressure is related to higher AF recurrence after ablation. In this context, the séifness of the left atrium, responsible for atrial pressure increase, is a contributing factor to the higher recurrence rates ${ }^{9,10}$. In a metaanalysis, left atrial stiffness was demonstrated as the most important predictor of arrhythmia recurrence after ablation, and it is suggested that an assessment of atrial stiffness, by non-invasive methods, be performed as a pre-ablation routine to identify patients with greater risk of recurrence ${ }^{10}$.

The diagnosis of SLAS is based on the presence of symptoms of dyspnea and right heart failure associated with deep $\mathrm{V}$ wave (wave $\mathrm{V}>10 \mathrm{mmHg}$ or wave $\mathrm{V}>$ mean value of the atrial pressure curve) and pulmonary hypertension (pulmonary pressure $\geq 25 \mathrm{mmHg}$ in rest or $\geq 30 \mathrm{mmHg}$ under stress) with preserved left ventricular function in the absence of significant mitral regurgitation and PV stenosis. Differential diagnosis mainly involves pulmonary thromboembolism, acute or subacute PV stenosis and left heart failure ${ }^{3,5,6}$.Some risk factors for the evolution with SLAS after AF ablation have been registered: diabetes mellitus, previous atrial scarring, smaller atrial diameter and obstructive sleep apnea syndrome ${ }^{2}$.

Non-invasive diagnostic methods have a complementary role in evaluating the syndrome. Echocardiography plays an important role in the diagnosis of SLAS, both in non-invasively estimating pulmonary arterial pressure and in excluding significant mitral regurgitation and PV stenosis.

Cardiac magnetic resonance imaging (CMR), in turn, is a relevant complementary tool for the assessment of atrial fibrosis through the delayed enhancement technique, which occurs after the ablative procedure, and is a factor that negatively influences the atrial diastolic function, inferring higher pressure atrial and aiding in the diagnosis of stiff atrium syndrome $\mathrm{e}^{2,11,12}$.

In a study by Khurram et al. ${ }^{13}$, an index to assess atrial diastolic function, known as the atrial stiffness index (ARF), and its stiffness have been described. This index is described as the pressure $(\mathrm{mmHg})$ necessary to induce an increase in atrial volume $(\mathrm{mL})$ and the pressure curve by invasive methods (during ablation) associated with the quantification of atrial volume by CMR. Based on this index, a stiff atrium presents less variation in its volume for the same pressure variation, compared to an atrium with greater compliance. This atrial dysfunction demonstrated by the ARF was worse at older ages, with previous ablations (due to fibrosis) and a higher recurrence rate.

Studies on the treatment of SLAS after RF ablation are very limited, and treatment with diuretics (such as furosemide) aims to reduce atrial preload and pressure, being effective for the resolution of the clinical picture. ${ }^{3,6}$. Interestingly, the response of pulmonary hypertension to diuretics in patients with SLAS appears to be better than the treatment of pulmonary hypertension caused by other diseases, suggesting that diuretics have better efficacy in treating SLAS and can be considered a first-line therapy. Some centers advocate the routine use of diuretics in the first days after the procedure, especially in patients with persistent or long-lasting persistent $\mathrm{AF}$, with the intention of avoiding the evolution of a symptomatic picture of systemic and/or pulmonary congestion.

\section{CONCLUSION}

SLAS is an uncommon syndrome after PAAF, however, with the progressive increase in the number of procedures, it is a diagnosis that needs to be remembered, as it has an important clinical impact on the patient, but with a good response to drug treatment (diuretics). The presence of atrial séifness, which causes an increase in left atrial pressures, is also related to a higher rate of arrhythmia recurrence and, therefore, it should be actively investigated, in some cases even before the ablative procedure. As a diagnostic method, cardiac magnetic resonance allows for an assessment directed at the left atrium, with the possibility of evaluating atrial systolic function, ARI and, mainly, the presence of atrial fibrosis by the late enhancement technique, an extremely relevant factor in the syndrome described. 


\section{AUTHOR'S CONTRIBUTION}

All authors contributed equally to the study

\section{DATA AVAILABILITY STATEMENT}

Data will be available upon request

\section{FUNDING}

This study has no financial support source.

\section{ACKNOWLEDGMENTS}

Not applicable.

\section{REFERENCES}

1. Hindricks G, Potpara T, Dagres N, Arbelo E, Bax J, Blomström-Lundqvist C, et al. 2020 ESC Guidelines for the diagnosis and management of atrial fibrillation developed in collaboration with the European Association for Cardio-Thoracic Surgery (EACTS). Eur Heart J. 2021;42(5):373-498. https://doi.org/10.1093/eurheartj/ehaa612

2. Gibson DN, Di Biase L, Mohanty P, Patel JD, Bai R, Sanchez J, et al. Stiff left atrial syndrome after catheter ablation for atrial fibrillation: Clinical characterization, prevalence, and predictors. Hear Rhythm. 2011;8(9):1364-71. http://dx.doi.org/10.1016/j. hrthm.2011.02.026

3. Yang Y, Liu Q, Wu Z, Li X, Xiao Y, Tu T, et al. Stiff left atrial syndrome: a complication undergoing radiofrequency catheter ablation for atrial fibrillation. J Cardiovasc Electrophysiol. 2016;27(7):884-9. https://doi.org/10.1111/jce.12966

4. Calkins H, Hindricks G, Cappato R, Kim YH, Saad EB, Aguinaga L, et al. 2017 HRS/EHRA/ECAS/APHRS/SOLAECE expert consensus statement on catheter and surgical ablation of atrial fibrillation. Hear Rhythm. 2017;14(10):e275-e444. http://dx.doi.org/10.1016/j. hrthm.2017.05.012

5. Calkins H, Hindricks G, Cappato R, Kim YH, Saad EB, Aguinaga L, et al. 2017 HRS/EHRA/ECAS/APHRS/SOLAECE expert consensus statement on catheter and surgical ablation of atrial fibrillation: Executive summary. J Arrhythmia. 2017;33(5):369-409. https://doi. org/10.1016/j.joa.2017.08.001

6. Gibson DN, Di Biase L, Mohanty P, Patel JD, Bai R, Sanchez J, et al. Stiff left atrial syndrome after catheter ablation for atrial fibrillation: Clinical characterization, prevalence, and predictors. Heart Rhythm. 2011;8(9):1364-71. https://doi.org/10.1016/j.hrthm.2011.02.026

7. Urey MA, Darden D, Stoller D, Drazner MH, Horn V, Sarma S, et al. Stiff Left atrial syndrome after multiple percutaneous catheter ablations: role for invasive hemodynamic exercise testing. Circ Hear Fail. 2017;10(5):e003885. https://doi.org/10.1161/ circheartfailure.117.003885

8. Correia ET de O, Barbetta LMDS, da Silva OMP, Mesquita ET. Left atrial stiffness: A predictor of atrial fibrillation recurrence after radiofrequency catheter ablation-a systematic review and meta-analysis. Arq Bras Cardiol. 2019;112(5):501-8. https://dx.doi. org/10.5935/abc.20190040

9. Park J, Joung B, Uhm JS, Shim CY, Hwang C, Hyoung Lee M, et al. High left atrial pressures are associated with advanced electroanatomical remodeling of left atrium and independent predictors for clinical recurrence of atrial fibrillation after catheter ablation. Heart Rhythm. 2014;11(6):953-60. https://doi.org/10.1016/j.hrthm.2014.03.009 
10. Gutterres DB, Derossi DA, Freitas JF, Peçanha LSP, Pimentel RC. Síndrome do átrio esquerdo rígido pós-ablação percutânea por catéter: relato de caso. 2018;97(6):589-93. https://doi.org/10.11606/issn.1679-9836.v97i6p589-593

11. Leite-Moreira AF, Oliveira SM, Marino P. Left atrial stiffness and its implications for cardiac function. Future Cardiol. 2007;3(2):17583. https://doi.org/10.2217/14796678.3.2.175

12. Wu TC. Left atrial stiffness, a marker of atrial cardiomyopathy, and atrial fibrillation - Relationships and predictors for procedure success after catheter ablation. Arq Bras Cardiol. 2019;112(5):509-10. https://doi.org/10.5935/abc.20190087

13. Khurram IM, Maqbool F, Berger RD, Marine JE, Spragg DD, Ashikaga H, et al. Association between left atrial stiffness index and atrial fibrillation recurrence in patients undergoing left atrial ablation. Circ Arrhythmia Electrophysiol. 2016;9(3):e003163. https://doi. org/10.1161/circep.115.003163 